\title{
Core-Substituted Naphthalene-Diimides (cNDI) and Related Derivatives: Versatile Scaffold for Supramolecular Assembly and Functional Materials
}

\author{
Anurag Mukherjee ${ }^{a}$ (D) \\ Suhrit Ghosh*a (iD) \\ a School of Applied and Interdisciplinary Sciences, Indian Association for the Cultivation \\ of Science, 2A and 2B Raja SC Mallick Road, Jadavpur, Kolkata 700032, India \\ psusg2@iacs.res.in
}

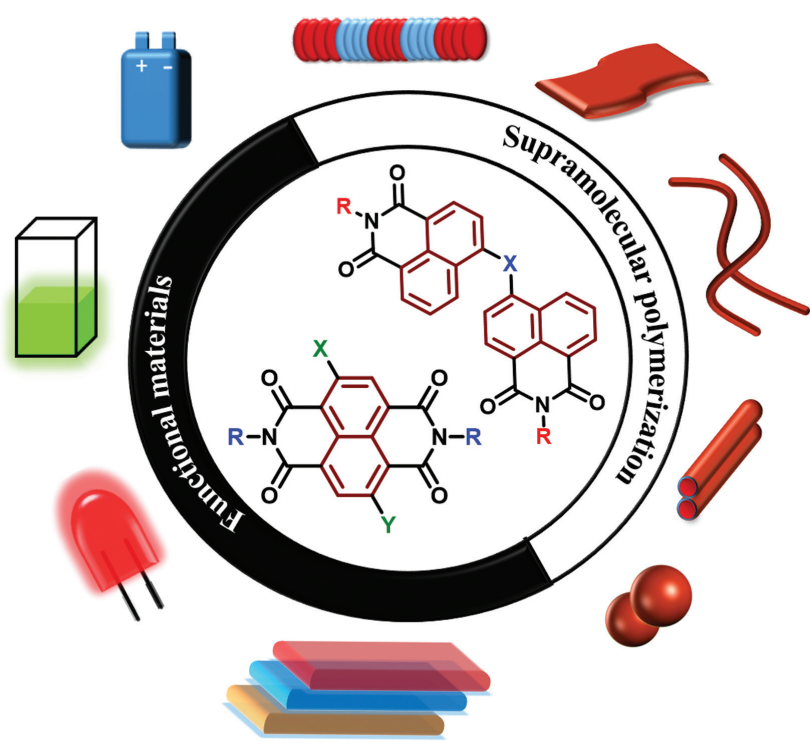

\section{Introduction}

$\pi$-Conjugated chromophores have emerged as promising candidates for designing functional materials via selfassembly with precise internal order and controllable morphology. ${ }^{1}$ Among various $\pi$-systems, naphthalenediimides (NDIs) and their core-substituted derivatives $(\mathrm{cNDI})^{2}$ have been used as versatile scaffolds for functional supramolecular systems because of their easy synthesis, wide-ranging functionalization and better solubility in common organic solvents compared to the higher homologues of the rylene-diimide family. ${ }^{3}$ NDIs are planar and neutral compounds with distinct redox activity. ${ }^{2}$ They exhibit high electron affinity, good charge carrier mobility, excellent thermal and oxidative stability and therefore had been used in various organic electronics applications. ${ }^{4}$

1,4,5,8-Naphthalenetetracarboxylic dianhydride (NDA) is the primary precursor for synthesizing different NDI derivatives. It can be readily functionalized at the anhydride position with different primary amines to get diimide derivatives, and depending on the nature of the substituents, a wide range of supramolecular building blocks can be produced which are suitable for self-assembly both in aqueous medium and hydrocarbon solvents. Under suitable reaction conditions, the aromatic ring of the NDI can also be substituted by different nucleophiles producing cNDIs 


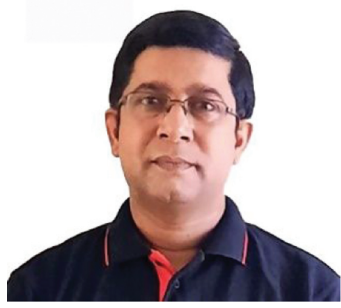

Suhrit Ghosh (born in 1976) did PhD (2000-2005) with Professor S. Ramakrishnan in the Department of Inorganic and Physical Chemistry, IISC Bangalore, India. Then he moved to the University of Massachusetts, Amherst, USA and worked as a postdoctoral research associate (2005-

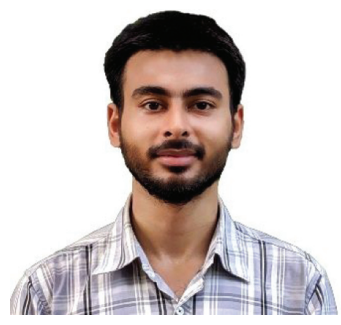

Anurag Mukherjee joined Indian Association for the Cultivation of Science, India in 2013 as an Integrated-PhD student. After obtaining his
2007) with Professor S. Thayumanavan. In 2007, he received the Alexander von Humboldt Postdoctoral Fellowship and moved to the group of Professor Frank Würthner in the University of Würzburg, Germany. In 2008 he joined as an Assistant Professor in the Polymer Science Unit of the
Indian Association for the Cultivation of Science (IACS), Kolkata, India. Currently he is a Senior Professor in the School of Applied and Interdisciplinary Science at IACS. His group's research interest includes Polymer Chemistry, Supramolecular Chemistry and Functional Materials.
(Figure 1), which differ significantly in terms of their photophysical and redox properties compared to the parent NDIs.

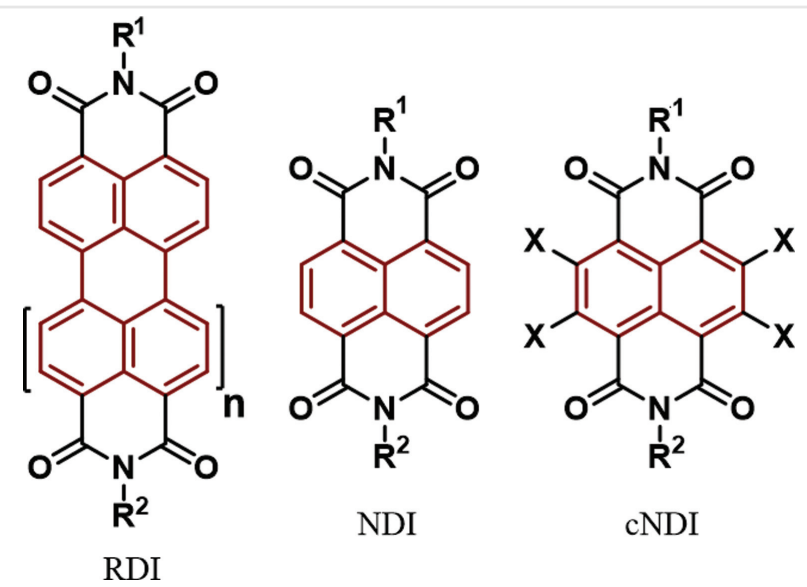

Figure 1 Structures of rylene-diimide (RDI), naphthalene-diimide (NDI) and core-substituted NDI (cNDI).

An early example of the synthesis of cNDIs was reported in 1937 by Vollmann and co-workers who demonstrated the incorporation of multiple chlorine atoms in the aromatic ring of the NDA under a harsh reaction condition involving fuming $\mathrm{HNO}_{3}{ }^{5}$ Later in 2002, Würthner and co-workers established a modified methodology for the synthesis of cNDIs with much improved yield. ${ }^{6}$ Further modifications of the synthetic procedure have been reported later by other groups where commercially available NDA could be brominated quantitatively using dibromo-cyanuric acid or master's degree in 2015 , he joined the research group of Professor Suhrit Ghosh for PhD. Since then, he has been working on the synthesis and supramolecular self-assembly of chromophore-containing polymers and small molecules. He has recently submitted his PhD thesis. 1,3-dibromo-5,5-dimethylhydantoin under acidic conditions (Figure 2). ${ }^{7}$ This halogenated intermediates can thereafter be reacted with various amines producing imide-substituted cNDIs and subsequent nucleophilic aromatic substitutions provide desired cNDI derivatives (Figure 2). Due to the weaker nature of the $\mathrm{C}-\mathrm{Br}$ bond, brominated-NDI intermediates undergo aromatic substitution reactions at a relatively faster rate compared to the chlorinated analogues. Würthner and co-workers

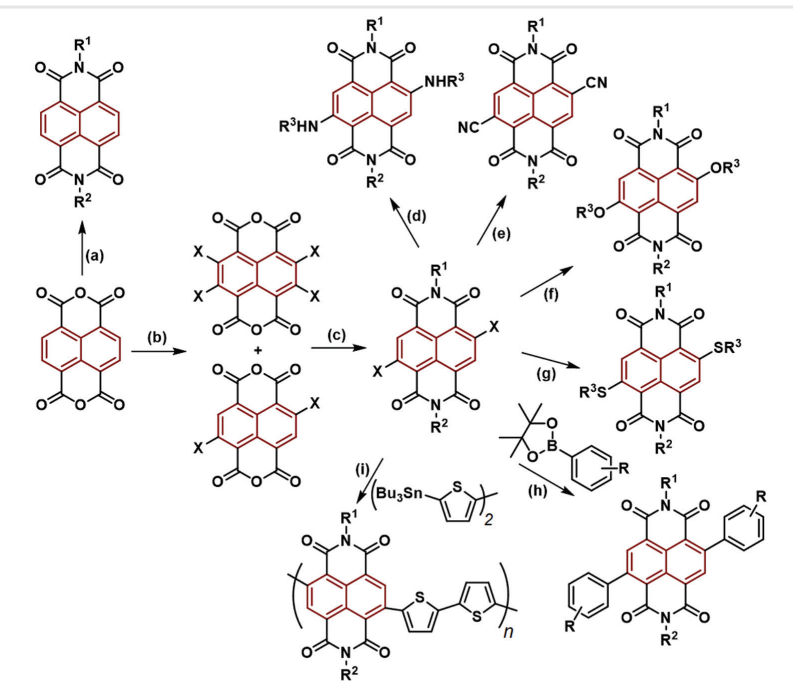

Reagent and conditions: (a) $\mathrm{R}^{1} \mathrm{NH}_{2}, \mathrm{R}^{2} \mathrm{NH}_{2}, \mathrm{DMF}, 140{ }^{\circ} \mathrm{C}$; (b) dibromocyanuric acid/ dibromohydantoin, $\mathrm{H}_{2} \mathrm{SO}_{4}$ (conc.), $55^{\circ} \mathrm{C}$; (c) $\mathrm{R}^{1} \mathrm{NH}_{2}, \mathrm{R}^{2} \mathrm{NH}_{2}, \mathrm{AcOH}, 100^{\circ} \mathrm{C}$; (d) $\mathrm{R}^{3} \mathrm{NH}_{2}$, rt; (e) CuCN, NMP, $100^{\circ} \mathrm{C}$; (f) $\mathrm{R}^{3} \mathrm{OH}, \mathrm{Na}, \mathrm{DMF}, 60^{\circ} \mathrm{C}$; (g) $\mathrm{R}^{3} \mathrm{SH}, \mathrm{K}_{2} \mathrm{CO}_{3}$, toluene, $100{ }^{\circ} \mathrm{C}$; (h) $\mathrm{Pd}\left(\mathrm{PPh}_{3}\right)_{4}$, toluene, $100{ }^{\circ} \mathrm{C}$; (i) $\mathrm{Pd}\left(\mathrm{PPh}_{3}\right)_{4}$, toluene, $100{ }^{\circ} \mathrm{C}$.

Figure 2 A generalized scheme for the synthesis of various derivatives based on NDI or CNDI scaffold. 
synthesized tetrabromonaphthalene-diimide and derivatised it with tetra-ethoxy, thioethyl, alkyl amine and ethylenediamines with considerable yields. ${ }^{7 c}$ Recently, Mukhopadhyay and co-workers successfully isolated tetracyano-naphthalene-diimide which is highly electron deficient in nature. ${ }^{7 d}$ The unstable molecule was further reduced using tetraphenylphosphonium iodide to obtain the stable planar radical anion. Proper stoichiometric balance and control during the ring substitution can also produce monobromo-substituted cNDIs. This can further be reacted with a different nucleophile to produce asymmetrically ring-substituted cNDI derivatives in moderate yields. ${ }^{7 a}$ Extended conjugated chromophores can be prepared starting from such halogen-substituted cNDIs by condensation with other $\pi$-systems using $\mathrm{C}-\mathrm{C}$ coupling reactions (Suzuki, Stille coupling and others), which significantly impacts the photophysical and electrochemical properties. There are also strategies to achieve core annulation of NDIs involving the formation of four-, fiveor six-membered ring annulations, bearing different heteroatomic and carbocyclic derivatives, which open up the possibility of designing supramolecular systems with tunable electronic characteristic, reduced band gaps and enhanced intermolecular overlap. ${ }^{8}$

NDIs are inherently $\pi$-acidic in nature, which makes them suitable for face-to-face $\pi$-stacking and transport of anions or electrons. Substitution at the aromatic core with suitable electron-withdrawing groups $\left(\mathrm{CN}\right.$ or $\left.\mathrm{NO}_{2}\right)$ can further increase the $\pi$-acidity. ${ }^{9}$ Matile and co-workers have explored the core-substitution-dependent variation of the electronic nature of several NDI-derivatives by calculating the quadrupole moment $\left(Q_{z z}\right)$ value, which was found to be $+18.6 \mathrm{~B}$ for unsubstituted NDI, confirming its $\pi$-acidic nature. A substitution with the electron-withdrawing cyano (CN) group in the NDI core enhances the $\pi$-acidity further with a $Q_{z z}$ value of +39.2 B. ${ }^{9 a}$ On the other hand, expectedly the $\pi$-acidity decreases with the introduction of electrondonating groups at the core. For example, the $Q_{z z}$ value of +2.3 B was reported for a cNDI with methyl amine substitution at the 2 - and 6 -positions. ${ }^{9 a}$

The extent of $\pi$-stacking interaction among the cNDI chromophores is often determined by the overall electron density around the aromatic ring and the resultant $\pi$-acidity thereof. cNDIs, substituted with electron-withdrawing substituents, exhibit stronger $\pi$-stacking by virtue of attractive dipole-moment-induced interactions and they are notable candidates for sensing, binding and transport of electrons, anions or other nucleophiles. A detailed discussion on the same could be found elsewhere. ${ }^{9 a}$ Stronger $\pi-$ stacking and face-to-face arrangements also facilitate the charge transport property of these cNDI derivatives via efficient intramolecular electronic coupling. However, the lesser requirement of the reorganisation energy, sometimes, assists an offset molecular arrangement to exhibit

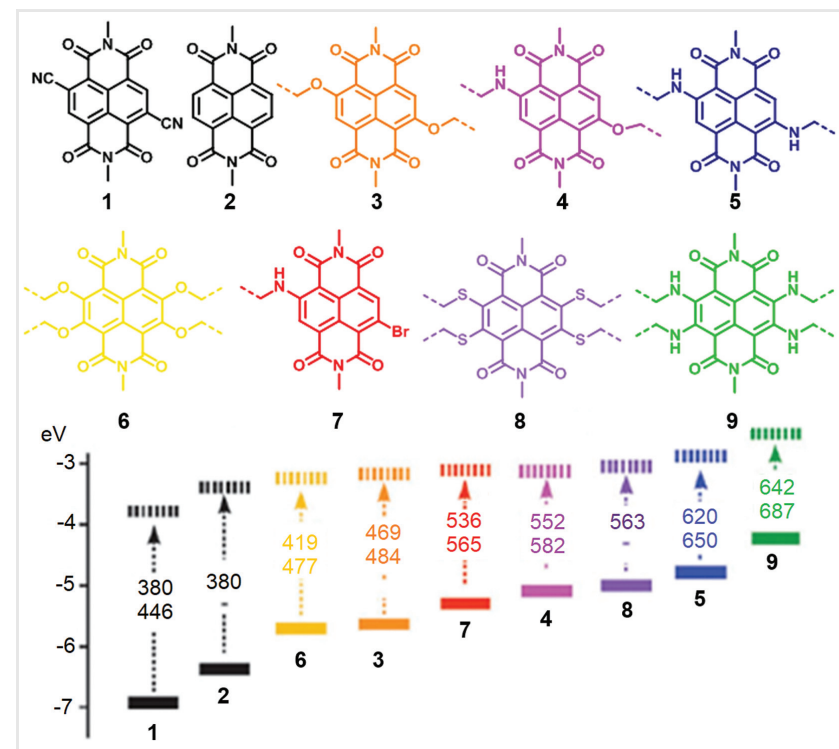

Figure 3 Molecular structures of NDI and cNDIs and corresponding HOMO (bold) and LUMO (dashed) energy levels (in eV) along with maximum absorption (top) and emission wavelengths (bottom, in nm). Adapted with permission from Ref. 9a. Copyright 2010 Royal Society of Chemistry.

higher charge transport values as it has the added advantage of preserved two-dimensional (2D) organization. ${ }^{10}$ Substitution with electron-donating or -withdrawing groups also changes the HOMO and LUMO energy levels, which gets reflected in the colour of the compounds and their redox behaviour (Figure 3). Imide-substituted NDIs exhibit $\pi-\pi^{*}$ transition bands in the range of $370-380 \mathrm{~nm}$ with vibrational fine structures which remain unaltered with the core substitution. ${ }^{9,11}$ In addition, cNDIs show an additional intramolecular charge transfer band in the visible range depending upon the nature of substitution (Figure 3).

Therefore, cNDIs appear to be highly versatile building blocks that can be functionalized either at the imide position and/or the aromatic rings, leading to the synthesis of wide-ranging derivatives with distinct optical properties. Depending on the structure of the building blocks, noncovalent interactions, such as $\pi-\pi$ stacking, hydrogen bonding, halogen bonding or solvophobic interactions can guide the supramolecular assembly or co-assembly with anther $\pi$-system. Early studies on the optical, redox and other photophysical properties of cNDIs as well as their supramolecular chemistry have been discussed in previously published review articles. ${ }^{9 a 11 c 2,7}$ Additionally, there are examples of cNDI-based donor-acceptor co-polymers with varied solid-state morphologies, which have been successfully implemented in $n$-channel and ambipolar organic field-effect transistors (OFETs) and those examples also have been discussed elsewhere. ${ }^{12}$ This short review 
discusses the recent development in this field with the primary focus on the specific opportunity that this versatile scaffold offers for unorthodox supramolecular structures and functions.

\section{General Supramolecular Assemblies of cNDI Derivatives}

Intrinsic fluorescent and electron-deficient nature of cNDI derivatives have been explored to constitute supramolecular polymers with elegant structures and important functions. Modarelli and co-workers reported bisterpyridine-functionalized cNDI derivatives (10 and 11, Figure 4) that formed extended supramolecular polymers in the presence of different divalent metal ions $\left(\mathrm{Ru}^{2+}, \mathrm{Fe}^{2+}\right.$ and $\left.\mathrm{Co}^{2+}\right) .{ }^{13}$ The typical high-emissive cNDI monomers exhibited negligible fluorescence in its metallo-supramolecular polymeric state due to electron transfer from the metal ions to the NDI moiety via the bridging ligands in the excited state. $^{13}$

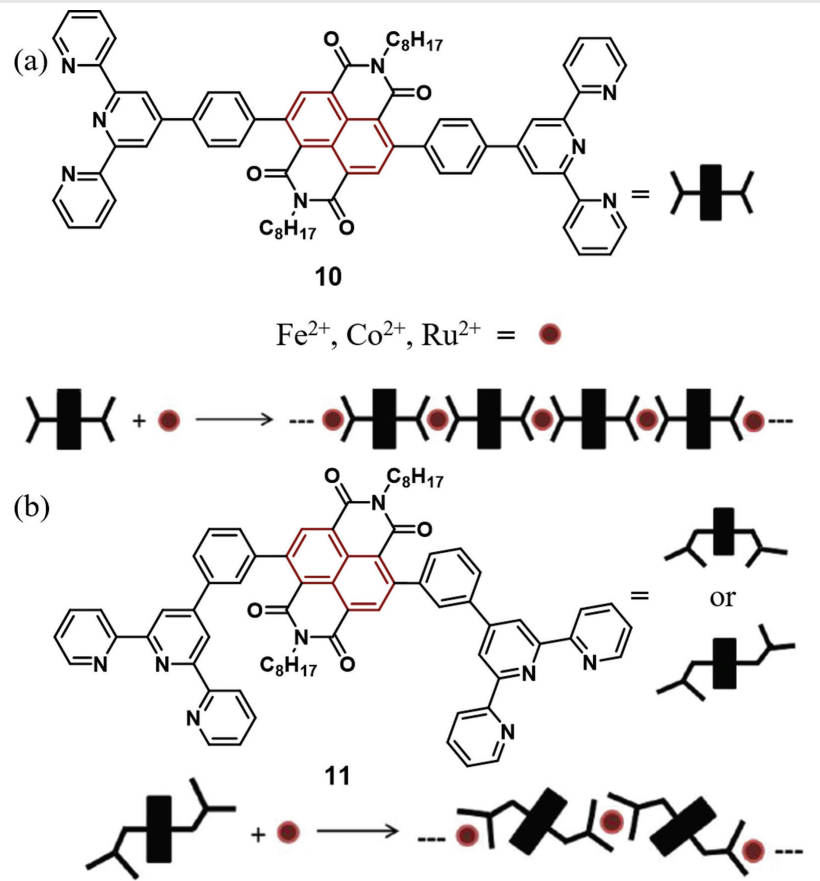

Figure 4 Metallo-supramolecular polymerization of bisterpyridylfunctionalized cNDI derivatives (10 and $\mathbf{1 1}$ ). Adapted with permission from Ref. 13. Copyright 2015 Royal Society of Chemistry.

George and co-workers demonstrated supramolecular polymerizations of two $\beta$-sheet forming peptide-tethered amphiphilic cNDI derivatives (12 and 13) and their $\mathrm{pH}-$ responsive behaviour (Figure 5$).{ }^{14}$ One of the cNDI building

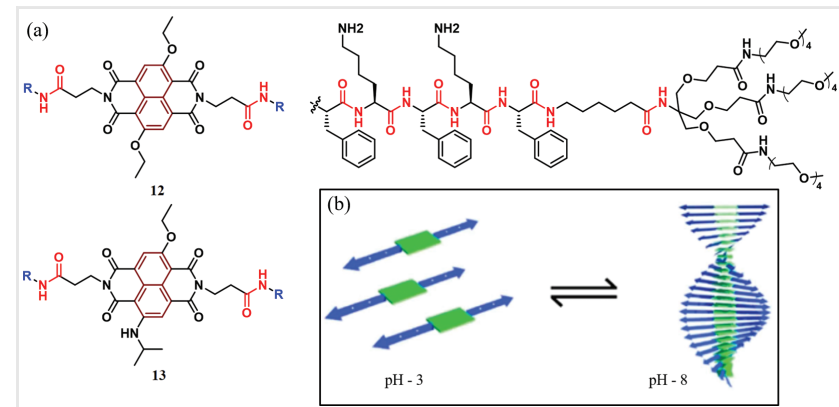

Figure 5 (a) Chemical structures of the peptide-appended cNDI derivatives and (b) model representation of the $\mathrm{pH}$-responsive supramolecular assembly. Adapted with permission from Ref. 14. Copyright 2020 Wiley-VCH.

blocks (12) was symmetrically functionalized with the diethoxy groups in the aromatic core, while the other one (13) was unsymmetrically substituted with isopropylamine and ethoxy groups. The difference in the nature of these hydrophobic substitutions in the cNDI moiety influenced the overall balance between the $\pi$-stacking, H-bonding, attractive hydrophobic interactions and repulsive sidechain interactions, operating in the dendritic peptide segments. This modulated the overall pH-responsive behaviour of the supramolecular assemblies of $\mathbf{1 2}$ and 13. cNDI derivative 12 showed $\mathrm{pH}$-responsive supramolecular polymerization, whereas the larger isopropyl moiety and slightly more electron-rich nature of the cNDI scaffold in $\mathbf{1 3}$ resulted in stable supramolecular polymers even in acidic medium. $^{14}$

Our group has reported a diamine-substituted D-A-D type cNDI gelator (14, Figure 6 ) that showed supramolecular polymerization by H-bonding among the imides and J-type $\pi$-stacking among the cNDI units, yielding micrometre-long nanotubes. ${ }^{15}$ Analysis of the cooling curves by UV-Vis spectroscopy revealed a highly co-operative nature of the supramolecular polymerization with the degree of polymerization in the range of 20-25. The elongation temperature $\left(T_{\mathrm{e}}\right)$ was found to be ranging from 321 to $336 \mathrm{~K}$ depending on the concentration, while the binding constant associated with the elongation step $\left(K_{\mathrm{e}}\right)$ was between 0.57 and $1.7 \times 10^{3} \mathrm{M}^{-1}$. The multilayer walls of the tubes, consisting of J-aggregated cNDI dyes, facilitated effective delocalization of the excited state, resulting in prolonged excited-state lifetimes extending beyond $100 \mu \mathrm{s} .{ }^{15}$

We recently reported the synthesis and self-assembly of a sulfur-substituted cNDI derivative (15, Figure 7) equipped with an amide functionality on either side. ${ }^{16}$ Supramolecular polymerization of $\mathbf{1 5}$ followed a nucleation-elongation pathway leading to the formation of J-aggregates with remarkable enhancement of the fluorescence quantum yield from 1\% in the monomeric state (in THF) to about 30\% in the self-assembled state (in decane). Time-correlated 

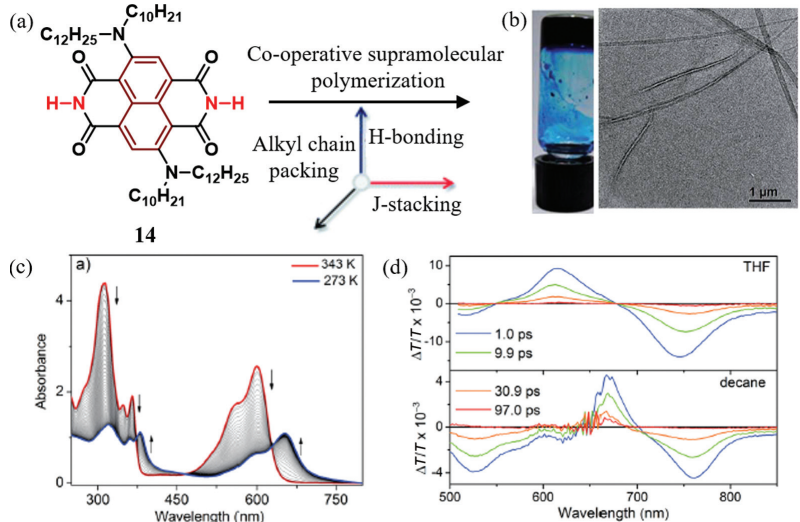

Figure 6 (a) Chemical structure of the diamine-substituted cNDI gelator and the associated (b) TEM images of the nanotubes in decane. (c) Temperature-dependent UV/Vis experiments in decane ( $c=1.75$ $\mathrm{mM}$ ), spectral changes are indicated by arrow. (d) Transient absorption spectra in THF and $n$-decane at different time delays $\left(\lambda_{\mathrm{ex}}=650 \mathrm{~nm}\right)$. Adapted from Ref. 15 published under a creative commons license (CC BY).
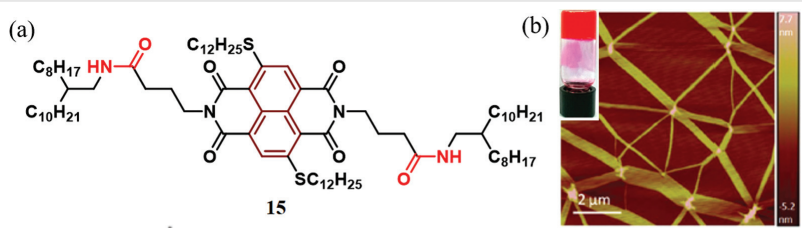

(c)
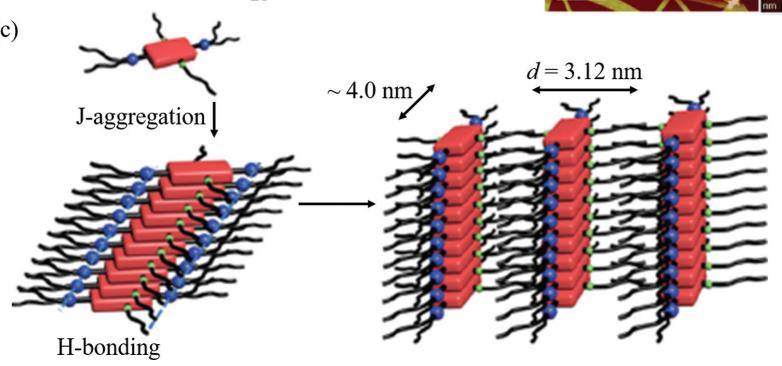

Figure 7 (a) Chemical structure and (b) nanotape-like assembly of sulfur-substituted cNDI gelator (15), inset image shows red-gel in decane. (c) Model representation of $\mathrm{H}$-bonding driven J-aggregation of 15. Adapted with permission from Ref. 16. Copyright 2016 Royal Society of Chemistry.

single photon counting revealed an average lifetime of $1.19 \mathrm{~ns}$ for the species in decane, which is notably large compared to that of $0.19 \mathrm{~ns}$ in THF. The rate constant for the non-radiative decay $\left(K_{\mathrm{nr}}=0.58 \mathrm{~ns}^{-1}\right.$ in decane $)$ in decane also exhibited a 16 -fold reduction with respect to the same in THF solution ( $K_{\mathrm{nr}}=8.98 \mathrm{~ns}^{-1}$ in THF) suggesting that the retardation of non-radiative decay pathways contributed to the enhanced fluorescence of the supramolecular polymers. It was proposed that the low-lying LUMO energy level of the sulphur-substituted cNDI in the J-aggregates inhibited inter-system crossing resulting in enhanced fluorescence. Compound 15 also behaved as a typical H-bonded gelator and formed robust organogel with exceptionally high thermal stability up to $90{ }^{\circ} \mathrm{C} .{ }^{16}$

Innovative molecular engineering around the cNDI scaffold provides ample opportunities to construct unorthodox building blocks enabling fabrication of higher order supramolecular assemblies including 2D sheets. Our group has reported a generalized design for 2D supramolecular assembly of an amphiphilic cNDI derivative (16). It was symmetrically substituted with two dodecane chains at the aromatic core, while the imide positions were functionalized with two hydrophilic wedges containing oligo-oxyethylene chains (Figure 8). ${ }^{17}$ It showed spontaneous selfassembly in aqueous medium with a rather low critical aggregation concentration of $1.5 \times 10^{-5} \mathrm{M}$, leading to the formation of ultrathin 2D sheets. Such entropy-favorable supramolecular assembly was driven by the $\pi$-stacking and crystalline packing of the hydrocarbon chains in two orthogonal directions. Differential scanning calorimetry trace showed the melting of the crystalline alkyl chains at $T>75{ }^{\circ} \mathrm{C}$, which destroyed the $2 \mathrm{D}$ assembly. Flashphotolysis time-resolved microwave conductivity (FPTRMC) studies revealed anisotropic conductivity in the $2 \mathrm{D}$ sheets with $\sim 3$ times larger mobility values along the parallel direction compared to that along the perpendicular direction. ${ }^{17}$
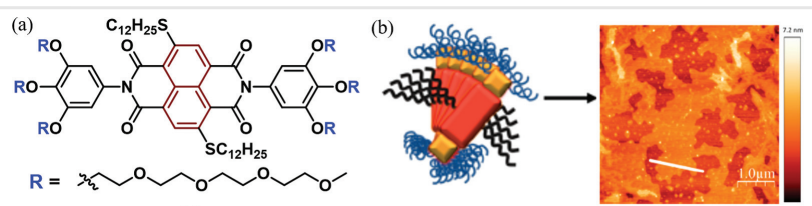

Figure 8 (a) Chemical structure of amphiphilic cNDI derivative (16) and (b) schematic representation of J-type stacking followed by AFM image of the ultrathin monodisperse nanosheet. Adapted with permission from Ref. 17. Copyright 2020 American Chemical Society.

George and co-workers recently reported redox-responsive supramolecular assembly of a $\beta$-alanine methyl ester appended c-NDI (17, Figure 9) having symmetrically substituted aromatic core with ethoxy groups. ${ }^{18}$ Compound 17 initially formed green-emitting vesicles in $\mathrm{pH} 7$ aqueous buffer (with 1\% DMSO) in which the cNDI showed Jaggregation. Under suitable conditions, it underwent twoelectron oxidation and the initially formed vesicular morphology converted reversibly into $2 \mathrm{D}$ sheets via a redox-dependent transient reconfiguration with concomitant colour change from green to red. ${ }^{18}$ 


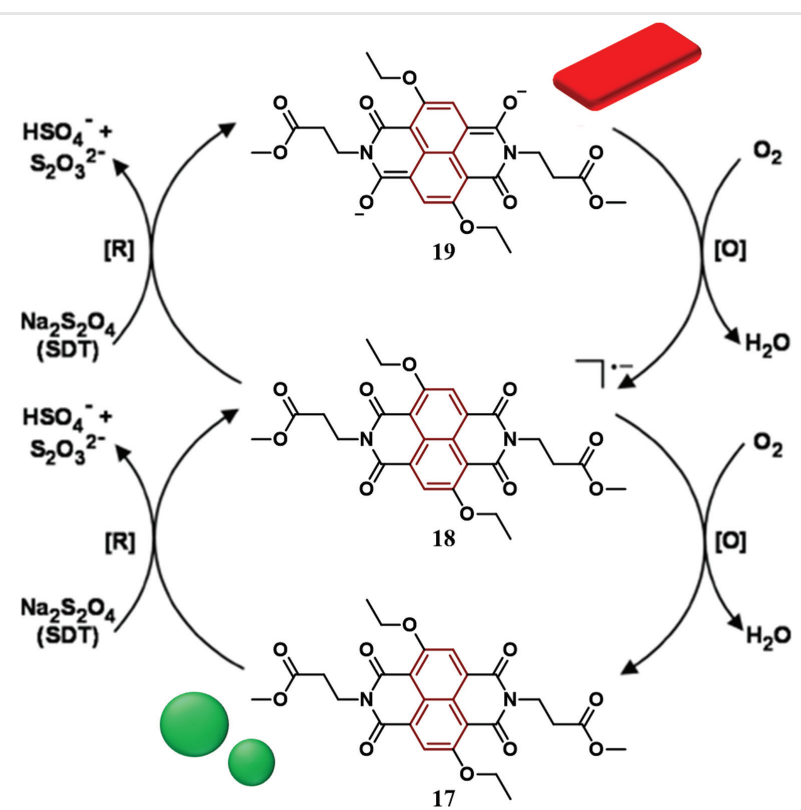

Figure 9 Chemical structure of methyl ester-appended cNDI derivative (17) and its redox-mediated morphology transition via radical anion (18) and dianion (19) formation.

\section{3. cNDI-Based Chiral Supramolecular Assemblies and Functional Materials}

Chirality of a supramolecularly assembled system is manifested by the formation of helical superstructures which are characterized by circular dichroism (CD) and it is distinguishable from its monomeric entity. Molecular chirality often influences the overall molecular packing and intramolecular interactions by suitable chiral recognition which may influence the performance of a functional material. ${ }^{19}$

George and co-workers have reported the chiralitydriven self-sorting of supramolecular polymers constituted by structurally similar core-substituted NDI donor (20) and acceptor derivatives $(\mathbf{2 1}, \mathbf{2 2})$ which were fluorescent in nature (Figure 10). ${ }^{20}$ These cNDIs were unsymmetrically substituted with ethoxy and isopropylamine groups and attached with a cyclic chiral linker such as (R,R)-BAC or (S,S)$B A C$ via imide substitution. The chiral recognition allowed stereoselective supramolecular polymerization via selfsorting with chirality control over the energy transfer process. However, energy transfer was observed to take place with higher efficiency in the co-assembled state which could be distinguished from the self-sorted state utilizing spectroscopic and microscopic methods. ${ }^{20}$

Nakashima and co-workers have utilized a chiral cNDIbased nanoplatform (23 and 24, Figure 11) for enantioselective recognition of chiral perylenediimide (PDI)-based acceptor molecules (25). ${ }^{21}$ To this quest, they synthesised

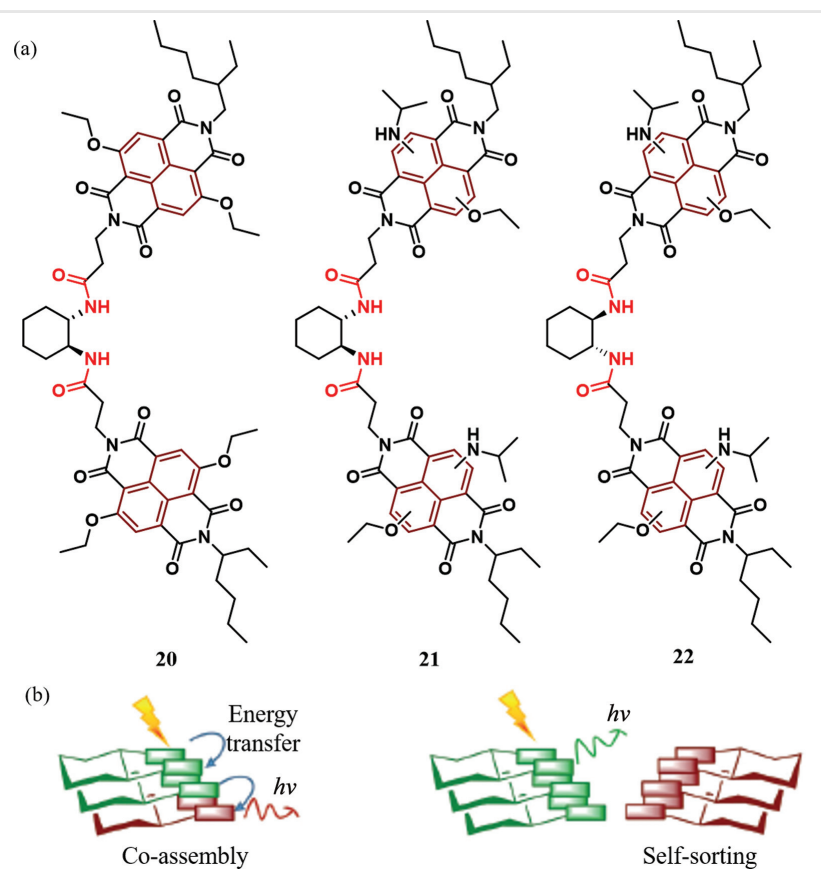

Figure 10 (a) Chemical structures of the donor (20) and acceptor (21 and 22) cNDIs. (b) Model representation of chirality-controlled selfassembly and light-harvesting process. Adapted with permission from Ref. 20. Copyright 2017 Wiley-VCH.

core-diethoxy-substituted bischromophoric cNDI building blocks attached covalently around a central cyclohexanediimide moiety in a chiral fashion (23 and $\mathbf{2 4}$ ). The compound showed H-bonding-driven self-assembly in methylcyclohexane $(\mathrm{MCH}) / \mathrm{CHCl}_{3}(19: 1)$ to produce chiral nanofibers with intense blue-green fluorescence. Introduction of a chiral PDIguest molecule (25) induced enantioselective recognition of the same by the cNDI-based chiral nanofibers with the morphology remaining mostly unaltered. The extent of the chiral recognition was successfully probed by measuring the efficiency of energy transfer from the cNDI donor molecules to the PDI acceptors revealing an enantioselectivity of almost $100 \%$. Circularly polarized luminescence (CPL) signal of the host cNDI system was also found to be sensitised

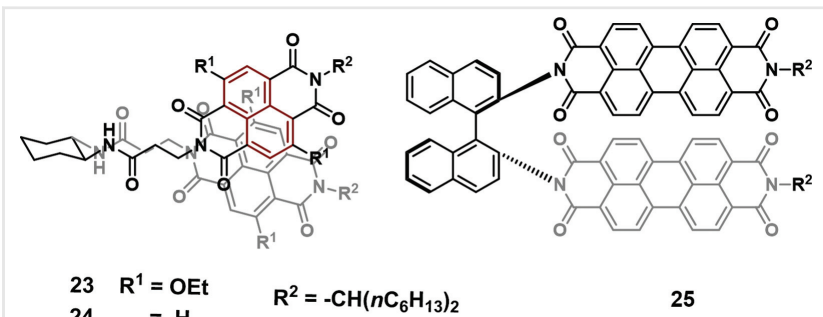

$$
\begin{aligned}
23 & R^{1} & =O E t & R^{2}=-\mathrm{CH}\left(n \mathrm{C}_{6} \mathrm{H}_{13}\right)_{2} \\
24 & =\mathrm{H} & & 25
\end{aligned}
$$

Figure 11 Chemical structures of the cNDI-based supramolecular nanoplatform (23 and 24) and the chiral PDI-guest (25). Adapted with permission from Ref. 21. Copyright 2019 Wiley-VCH. 
by the chiral guest incorporation as the wavelengths and sign of CPL bands exhibited notable deviations. ${ }^{21}$ The similar bischromophoric chiral cNDI building block (23, Figure 11) was explored for pressure-dependent binding and release of chiral PDI-guest molecules. The overall phenomena resemble that of protein bindings. The twisted arrangements of the cNDI units around the cyclohexyl-1, 2-diimide framework created suitable binding pockets with possible $\pi$-stacking interaction sites. The pressure-dependent association constant $\left(K_{\mathrm{a}}\right)$ could be estimated by examining the extent of fluorescence quenching of the host cNDI blocks in the presence of the acceptor PDI-blocks using the Stern-Volmer equation. The $K_{\mathrm{a}}$ value increased gradually to $4.9 \times 10^{-5} \mathrm{M}^{-1}$ at $50 \mathrm{MPa}$ followed by a steady decrease to $1.6 \times 10^{-5} \mathrm{M}^{-1}$ at 300 MPa. $^{22}$

This cNDI building block (23, Figure 11) was further examined to understand the impact of optical purity upon the organization and energy migration along the chromophoric stacks. Enantiomerically pure compound 23 showed H-bonding-driven supramolecular polymerization to produce well-defined straight nanofibers, whereas the racemic solution consisted of curved and entangled fibers. The emission efficiency of these nanofibers was found to be dependent upon the enantiomeric excess (ee) values which also influenced the chiral recognition and energy migration efficiency in presence of the chiral PDI-acceptors (25). ${ }^{23}$

Our group explored the supramolecular polymerization of a sulfur-substituted cNDI derivative equipped with chiral side chains (26, Figure 12). ${ }^{24}$ The molecule followed a cooperative supramolecular polymerization pathway leading to the formation of fluorescent J-aggregates with a helical fibrillar morphology. Chirotopic properties of the molecule could be manifested from the CD spectroscopy and these chiral supramolecular polymers also exhibited prominent CPL signal with a rather high luminescence dissymmetry factor $\left(g_{\text {lum }}\right)$ of $4.6 \times 10^{-2}$. Utilization of $\mathbf{2 6}$ as the chiral seed for initiating seeded supramolecular polymerization from a structurally similar achiral cNDI derivative (15) was also studied. Interestingly, no sergeantand-soldier effect could be noticed when a mixture of $\mathbf{2 6}$ (chiral) with 15 (achiral) (compound 26/compound $15=1: 9$ ) was heated and slowly cooled to room temperature in decane. However, when a monomeric solution of $\mathbf{1 5}$ in THF was injected to pre-formed chiral seed solution of $\mathbf{2 6}$ in decane, a prominent $C D$ signal appeared indicating chiral amplification by the sergeant-and-soldier principle. This resulted in an induced CPL with $g_{\text {lum }}$ of $2.0 \times 10^{-2}$ from mostly (>98.5\%) achiral building blocks. ${ }^{24}$

In a related report, supramolecular polymerization of this hydrophobic cNDI derivative (26) was studied in a confined aqueous medium (Figure 13). ${ }^{25}$ Encapsulation of $\mathbf{2 6}$ in the polyethylene glycol (PEG-2000)-based DSPE-lipid (27) allowed the formation of nanorods having lengths in the range of $170-230 \mathrm{~nm}$ and widths of $\sim 40-60 \mathrm{~nm}$. Dimensions of the (a)

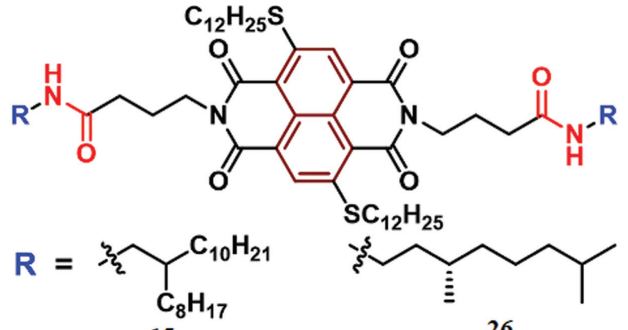

(b)

15

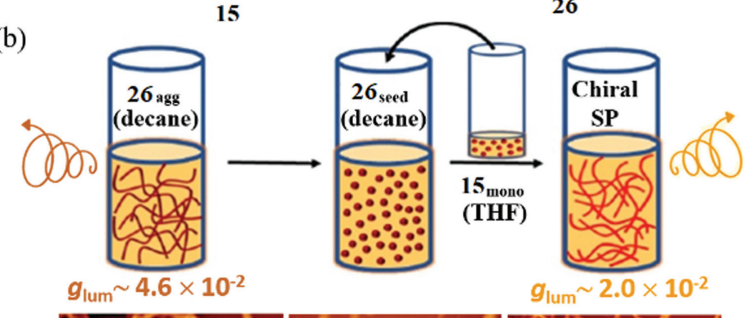

26

(c)
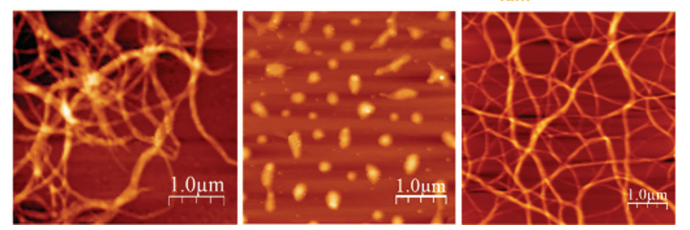

Figure 12 a) Chemical structures of the achiral (15) and chiral (26) cNDI derivatives. (b) Schematic representation of the seeded supramolecular polymerization and (c) corresponding AFM images of each component; the glum values indicate CPL dissymmetry factor of the respective supramolecular polymers. Adapted with permission from Ref. 24. Copyright 2020 Wiley-VCH.

lipid-encased cNDI nanorods could be tuned by varying the monomer/lipid ratio. UV-Vis studies revealed identical Jaggregation of $\mathbf{2 6}$ in the confined environment similar to that observed in the bulk hydrocarbon medium. Importantly, a remarkable confinement effect was noticed on the stability of the J-aggregate in this case as no thermal disassembly was observed up to $90^{\circ} \mathrm{C}$ in contrast to the complete disassembly of

(a)
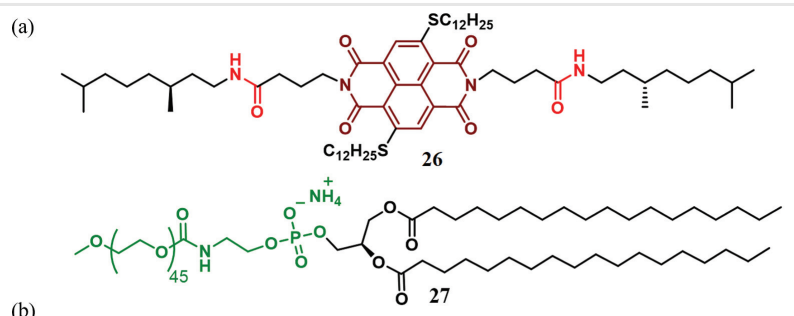

(b)

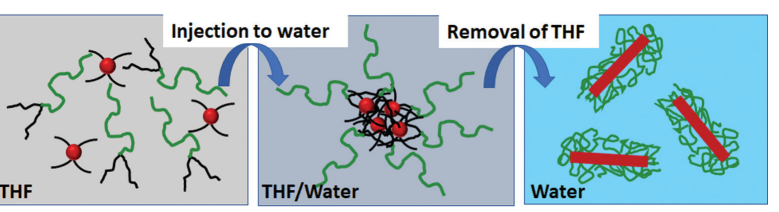

Figure 13 (a) Chemical structures and (b) supramolecular polymerization of $\mathbf{2 6}$ in confined environment. Adapted with permission from Ref. 25. Copyright 2020 Royal Society of Chemistry. 

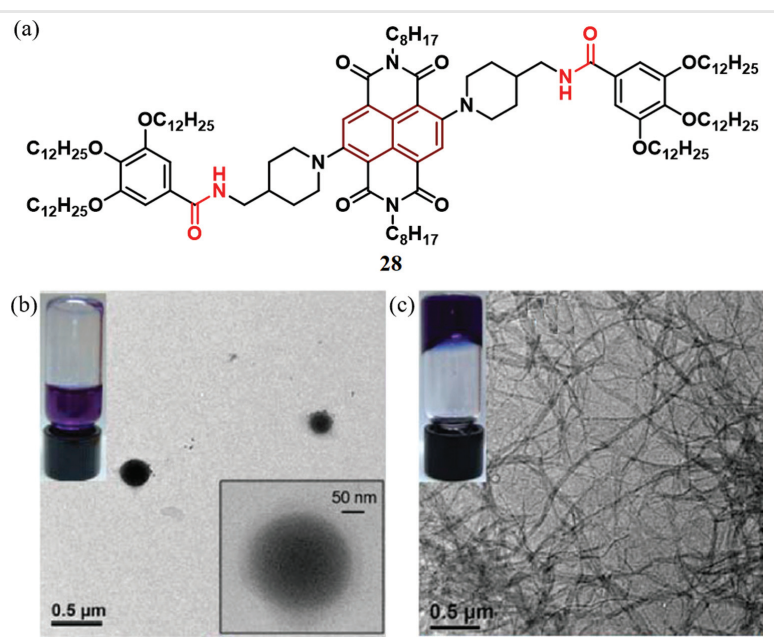

Figure 14 (a) Chemical structure of amide-functionalized cNDI molecule (28). (b) TEM images of $\mathbf{2 8}$ in cyclic (MCH) and linear (decane) hydrocarbon solvents and corresponding sol/gel images in the inset. Adapted from Ref. 28 published under a creative commons license (CC BY).

the same J-aggregates in decane above $60{ }^{\circ} \mathrm{C}$. Furthermore, the confined supramolecular polymer showed significant enhancement in the fluorescence quantum yield (50\%) compared to the already high value of $30 \%$ for the bulk J-aggregates in decane. Likewise, the CPL studies of the chiral J-aggregates in the confined environment showed a two-fold increase in the $g_{\text {lum }}$ values $\left(7.5 \times 10^{-2}\right)$ compared to that in decane. ${ }^{25}$

\section{Controlled Supramolecular Polymerization with cNDI Derivatives}

Controlled supramolecular polymerization (CSP) asks for congruent structural precision and monodispersity in the self-organisation of smaller building blocks that gets reflected in the resultant morphology which is often predictable. ${ }^{26}$ In the recent past, a large number of reports have elucidated CSP from different $\pi$-systems. ${ }^{26,27}$ Among them, cNDI is an attractive scaffold because depending on the nature of the substitutions, they exhibit different optical properties which permits probing their sequence-defined co-assembly by spectroscopic techniques. For example, in $\mathrm{H}$-bonding-driven supramolecular copolymerization supramolecular copolymerization studies, often it is desirable that the two different monomers are not significantly different in shape or size but exhibit different emission/chirality so that the copolymerization can be probed by fluorescence microscopy or CD spectroscopy. cNDI derivatives provide such opportunities because by different core-substitutions their photophysical properties change significantly, but the location of the H-bonding functional groups (amide or others) attached to the imide position does not change with respect to each other. Therefore, two different cNDI derivatives still can copolymerize and if one of them is chiral and other is achiral, such copolymerization can be probed by induced chirality in $C D$ spectroscopy. Likewise, their different emission properties allow probing such copolymerization by fluorescence microscopy. Any two different chromophores will not have these advantages as their difference in shape and size may inhibit copolymerization in a predictable manner.

Supramolecular polymerization of a diamine-substituted cNDI derivative (28, Figure 14) was explored by our group which exhibited H-bonding driven J-aggregation. ${ }^{28}$ The intramolecular electron transfer dynamics in such Jaggregates was probed using transient absorption spectroscopy, revealing contrasting behaviours between the monomeric and self-assembled states. For the monomeric dye in THF, a sub-ps intramolecular electron transfer, followed by a fast recombination, was noticed. A remarkable enhancement of the excited state lifetime by more than 1 order of magnitude could be observed for the J-aggregated supramolecular polymers in $\mathrm{MCH}$, which can be attributed to the effective delocalization of the charge-separated states. ${ }^{28}$ Similar aggregation and photophysical properties of $\mathbf{2 8}$ could be noticed in few other hydrocarbon solvents. However, the shape of the solvents imparted a profound effect on the aggregated morphology. In linear hydrocarbons such as $n$-hexane, n-octane, $n$-decane or $n$ dodecane, macroscopic gelation was observed and the formation of entangled fibrillar network was evident from the transmission electron microscopy images. Contrastingly, in cyclic hydrocarbons like $\mathrm{MCH}$ or cyclohexane, it produced spherical particles with the diameter in the range of 135-150 nm (Figure 15). This indicated a significant

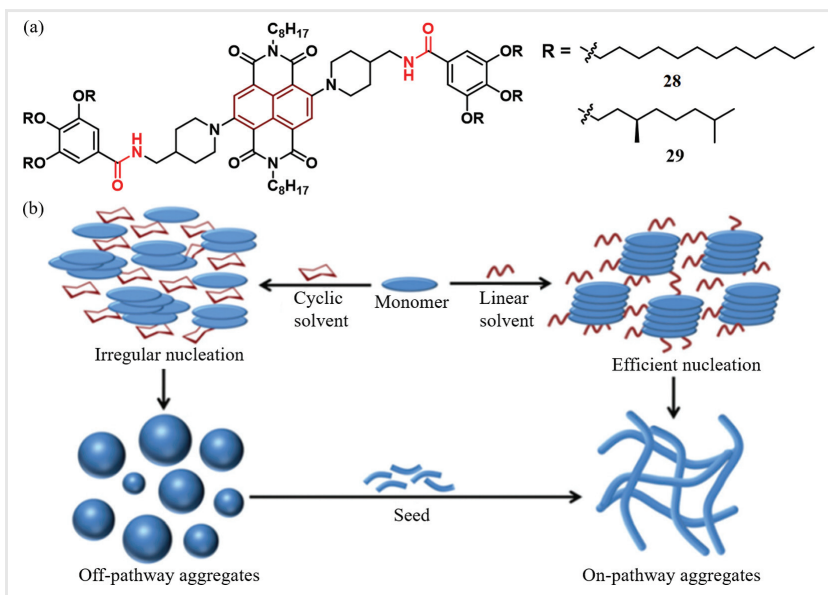

Figure 15 (a) Chemical structures of achiral (28) and chiral (29) cNDI derivatives and (b) schematic representation of solvent-geometryregulated pathway selection and seeded supramolecular assembly. Adapted with permission from Ref. 29. Copyright 2017 Wiley VCH. 
shape effect of the solvent on the supramolecular polymerization, which was subsequently investigated in more detail. ${ }^{29}$

Mechanistic investigation utilizing UV-Vis spectroscopy studies revealed a cooperative supramolecular polymerization occurring in the linear hydrocarbons which could be attributed to an effective nucleation due to the shape matching of the solvent molecules with the peripheral alkyl chains of the monomer. In contrast, the supramolecular polymerization in cyclic solvents like $\mathrm{MCH}$ followed an illdefined pathway leading to the formation of spherical aggregates. These particle-like aggregates in $\mathrm{MCH}$ could be identified as kinetically trapped species which were used as the monomer reservoir for seeded supramolecular polymerization. Seeds were obtained by sonication-induced fragmentation of the fibrillar structures produced in the linear hydrocarbon solvents. CSP using seeds of chiral cNDI derivative (29) were investigated, which yielded helical supramolecular polymers with tunable size from offpathway aggregates of both chiral (29) and achiral (28) cNDIs. ${ }^{30}$

George and co-workers recently reported two cholesterol-appended cNDI derivatives (Figure 16) with the NDI rings being symmetrically substituted with either thioethyl (30) or ethoxy groups (31). ${ }^{31}$ Such core-substitutions resulted in the emission of different colours (red and green, respectively) in these molecules, while the cholesterol group invoked long-range dipole-dipole interactions which contributed to the CSP. These cNDI molecules were tested for multicomponent supramolecular copolymerization to construct self-sorted supramolecular homopolymers, random (statistical) supramolecular co-polymers, alternating supramolecular copolymers and complex supramolecular block copolymers by optimization of the exchange dynamics and preferential intermolecular interactions (Figure 16). Supramolecular polymerization of the monomers followed distinct pathway complexity which could be explored for trapping suitable metastable states while manipulating the thermodynamic and kinetic routes during the two-component sequence CSP. The distinct optical properties and red/green fluorescence of $\mathbf{3 0}$ and 31, respectively, were utilized to probe the monomer exchange dynamics in their stacked organization and could be visualized by super resolution microscopy. Simultaneously, spectroscopic evidence was obtained by measuring the $\mathrm{CD}$ signals induced from the chiral side chains, which was further supported by mechanistic evidence from molecular dynamics simulations. $^{31}$

Subsequently, the same group reported thermodynamically controlled supramolecular block copolymers of fluorescent cNDI derivatives and corresponding functional organic heterostructures. The two chromophores, under discussion, showed complimentary optical behaviours, hence heading to axial organic heterostructures with

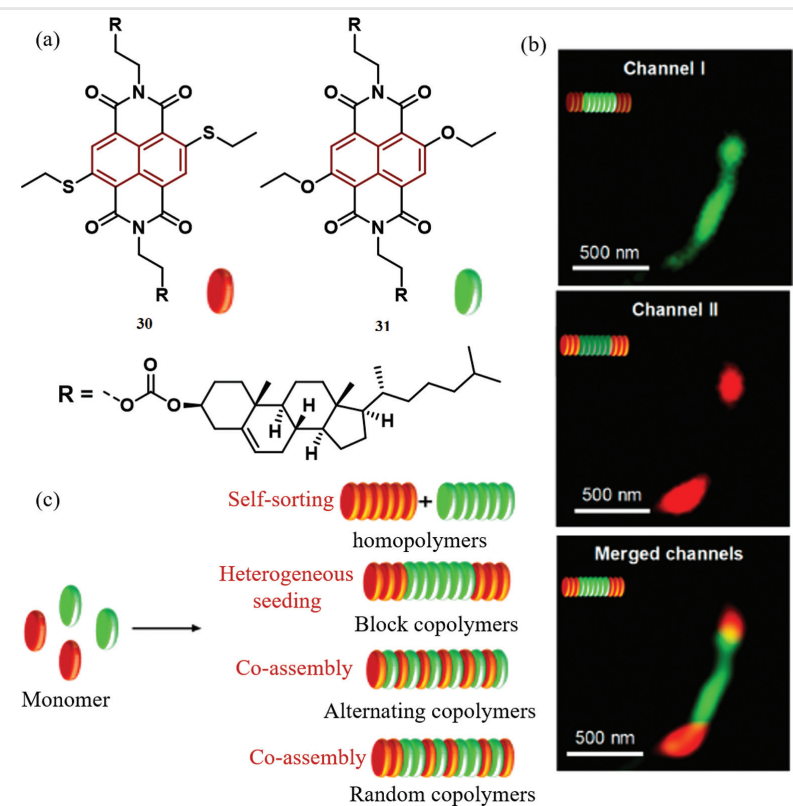

Figure 16 (a) Chemical structures of $\mathbf{3 0}$ and 31. (b) SIM images depicting supramolecular block copolymers. (c) Schematic representation of the multicomponent supramolecular copolymerization. Adapted with permission from Ref. 31. Copyright 2020 American Chemical Society.

light-harvesting properties (Figure 17). ${ }^{32}$ To this quest, the authors have utilized ethoxy $\left(\mathbf{3 2}, \mathrm{OC}_{2} \mathrm{H}_{5}\right)$ and pentanethiol (33, $\left.\mathrm{SC}_{5} \mathrm{H}_{11}\right)$-substituted cNDI derivatives with green and red florescence, respectively, and attached them with chiral (S,S)-trans-1,2-bis(amido)-cyclohexane motifs (Figure 17). These cNDI building blocks showed supramolecular polymerization via the nucleation-elongation pathway and the formation of the block copolymers could be probed by CD spectroscopy. The block copolymers exhibited a narrow

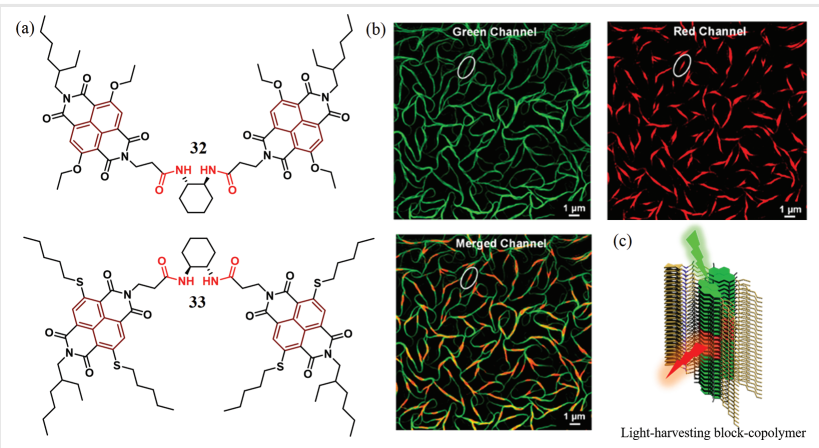

Figure 17 (a) Chemical structures of cNDI monomers participating in the formation of supramolecular axial heterostructure; (b) SIM microscopy images depicting the formation of supramolecular block copolymers and (c) corresponding schematic representation of the light-harvesting heterostructure. Adapted with permission from Ref. 32. Copyright 2020 American Chemical Society. 
polydispersity index of 1.1 and the multiblock nanostructures could be visualized using a structured illumination-based microscopy technique. ${ }^{32}$

\section{Dimeric Naphthalimide-Based Building Blocks}

Unlike NDIs, naphthalimides or naphthalene monoimides (NMIs) are functionalized with a single-electronwithdrawing imide segment attached at the 1,8-position of the naphthalene ring. Similar to cNDIs, core-substituted naphthalimides (cNMIs) also behave as electron-deficient $\pi$-systems and they often found applications in organic photovoltaics, OFET transistors and memory devices because of their tunable absorption spectra with high extinction coefficients, effortless functionalization, amenable for photo-stability and ease of device fabrication. ${ }^{33}$ Such cNMI derivatives also have been used for supramolecular assemblies, ${ }^{34}$ which is out of the scope of this particular article. These cNMI units, if connected to two arms of an electron-rich moiety, produce A-D-A-type systems with distinct photophysical properties. This is a particularly interesting class of $\pi$-systems because in one hand their photophysical properties can be tuned by varying the conjugation length/nature of the linker chromophore while on the other hand, the terminal imide groups can be suitably functionalized to enhance the structural diversity of the supramolecular building blocks.

Our group has recently reported the synthesis of ambipolar $\pi$-conjugated A-D-A type chromophores (34 and $\mathbf{3 5}$, Figure 18 ) by the covalent attachment of

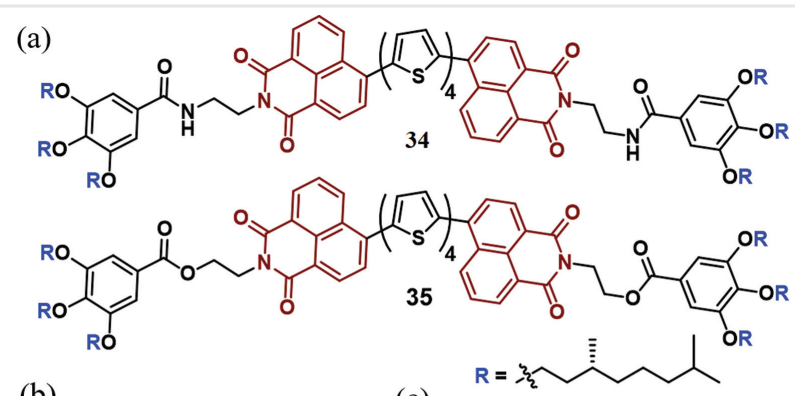

(b)

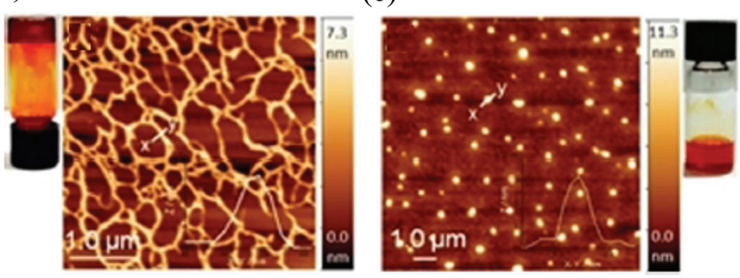

Figure 18 (a) Chemical structures of the ambipolar r-conjugated chromophores and (b and c) respective solution pictures and AFM images in MCH. Adapted with permission from Ref. 35. Copyright 2019 Wiley-VCH. quaterthiophene with NMI units which were further connected to trialkoxy benzamide wedges. ${ }^{35}$ Compound 34 revealed $\mathrm{H}$-bonding-assisted supramolecular polymerization in MCH leading to the formation of entangled fibrillar morphology and gelation at higher concentration. The extended conjugation lowered the HOMO-LUMO gap significantly $(\Delta E \sim 2.1 \mathrm{eV})$ and the H-aggregated supramolecular assembly significantly enhanced electrical conductivity, which was in the range of $2 \times 10^{-2}{\mathrm{~S} . \mathrm{cm}^{-1}}^{\text {. However, }}$ the ester-based control molecule (35) lacked any H-bonding interaction and thus produced near-spherical particles. ${ }^{35}$

Favereau, Srebro-Hooper, Crassous and co-workers investigated the solvent polarity-dependent modulation of CPL of $\pi$-helical push-pull systems (Figure 19).

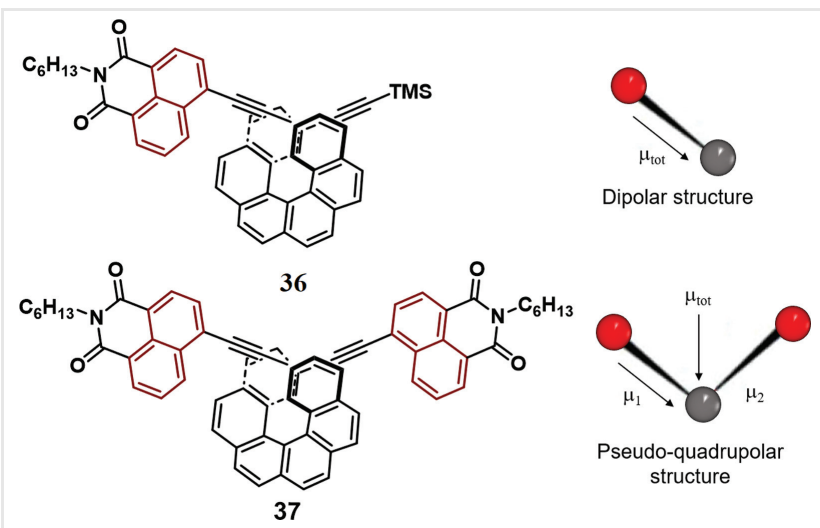

Figure 19 Chemical structures of the AD (36) and ADA (37) type pushpull chromophore and plausible direction of resultant dipole moment of them. Adapted from Ref. 36 published under a creative commons license (CC BY).

Helicene-conjugated enantiopure A-D (36, Figure 19) and A-D-A-type (37, Figure 19) naphthalimide derivatives were synthesized which exhibited high quantum yields (up to $80 \%$ ) and intense CPL signals ( $g_{\text {lum }} \sim 10^{-3}$ and $10^{-2}$, respectively) in cyclohexane. Compound $\mathbf{3 6}$ was assigned to be mostly dipolar in nature, whereas $\mathbf{3 7}$, as of its unique electronic conjugation, behaved as a pseudo-quadrupolar system. Interestingly, compound 37 exhibited a detrimental effect of solvent polarity on the CPL intensity as changing the solvent from nonpolar cyclohexane $\left(g_{\text {lum }} \sim 9.5 \times 10^{-3}\right)$ to polar DMF ( $g_{\text {lum }} \sim 3 \times 10^{-3}$ ) resulted in a less intense broad CPL spectrum with a low chiral dissymmetry factor. However, CPL intensity for compound $37\left(g_{\text {lum }} \sim 2 \times 10^{-3}\right)$ remained mostly unaltered in all the tested solvents. This effect of solvent polarity on the excited-state chiroptical properties of $\mathbf{3 7}$ was attributed to the change in the symmetry of the emitting $S_{1}$-state along with the truncated excitonic coupling among the individual donor-acceptor intra-molecular charge transfer transitions in polar solvents. $^{36}$ 
Fukui, Shinokubo and co-workers synthesized a nitrogen-bridged naphthalene-monoimide dimer (38) with green emission in solid-phase self-assembly with a quantum yield of $50 \%$. The intense emission of $\mathbf{3 8}$ was attributed to the favourable packing pattern guided by $\mathrm{CH} / \pi$ interaction as revealed from X-ray diffraction (Figure 20). ${ }^{37}$

(a)
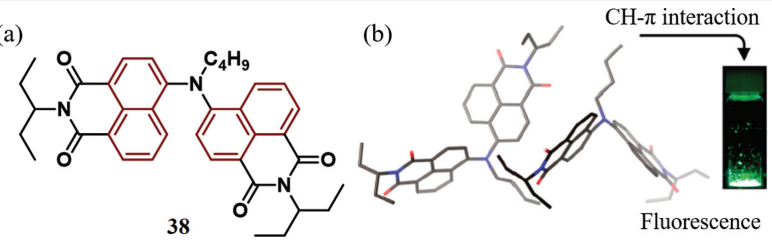

Figure 20 (a) Chemical structure of AIE capable NMI-dimer (38) and (b) corresponding packing pattern revealing $\mathrm{CH} / \pi$ interaction. Adapted with permission from Ref. 37. Copyright 2019 American Chemical Society.

\section{Conclusions and Outlook}

In this article, we have described relevant recent examples on the functional supramolecular assemblies based on core-substituted naphthalene-imide scaffold. We have exemplified cNDI-based building blocks for supramolecular polymerization to produce enticing nanostructures with excellent fluorescence, $\mathrm{CPL}$ or stimuli responsive behaviour. In another section, cNDI derivatives have been highlighted for CSP with structural precision. Finally, supramolecular assembly of dimeric core-substituted naphthalene-monoimide derivatives have been discussed. NDI and related monoimide derivatives provide the scope for adequate structural engineering with tunable morphologies and optoelectronic properties with easy device fabrication possibilities and thus may be considered to be prospective candidates for creating diverse supramolecular materials. Independent functionalization at the imide positions and in the aromatic ring provides suitable opportunities in terms of structural diversity and functional utilities of this class of molecules. Although cNDI derivatives have been studied in the context of optoelectronic $^{38}$ and other applications, ${ }^{39}$ their supramolecular polymerization has not been fully explored yet, compared to the vast literature on the supramolecular assembly of NDI derivatives without any core substitutions. It also needs to be understood how different core substitution affects the $\pi$-stacking strength in this family of chromophores. A-D-A or D-A-D type $\pi$-systems containing NDI or NMI units as the acceptor motifs are even less known. They may turn out to be an important addition in the emerging field of ambipolar $\pi$-systems ${ }^{40}$ for various optoelectronic applications.

\section{Funding Information}

S.G. thanks Science and Engineering Research Board (Project No: CRG/2020/002395), India for funding.

\section{Acknowledgment}

A.M. thanks IACS for a research fellowship.

\section{References}

(1) (a) Aida, T.; Meijer, E. W.; Stupp, S. I. Science 2012, 335, 813. (b) Hoeben, F. J. M.; Jonkheijm, P.; Meijer, E. W.; Schenning, A. P. H. J. Chem. Rev. 2005, 105, 1491. (c) Babu, S. S.; Praveen, V. K.; Ajayaghosh, A. Chem. Rev. 2014, 114, 1973. (d) Würthner, F.; Saha-Möller, C. R.; Fimmel, B.; Ogi, S.; Leowanawat, P.; Schmidt, D. Chem. Rev. 2016, 116, 962. (e) Das, A.; Ghosh, S. Angew. Chem. Int. Ed. 2014, 53, 2038. (f) Yagai, S.; Kitamoto, Y.; Datta, S.; Adhikari, B. Acc. Chem. Res. 2019, 52, 1325. (g) Rest, C.; Kandanelli, R.; Fernández, G. Chem. Soc. Rev. 2015, 44, 2543. (h) Kumar, M.; Rao, K. V.; George, S. J. Phys. Chem. Chem. Phys. 2014, 16, 1300. (i) Dorca, Y.; Mattern, J.; Fernández, G.; Sánchez, L. Isr. J. Chem. 2019, 59, 869.

(2) (a) Dan Pantos, G. Naphthalenediimide and its Congeners: From Molecules to Materials. Royal Society of Chemistry: Cambridge, 2017. (b) Al Kobaisi, M.; Bhosale, S. V.; Latham, K.; Raynor, A. M.; Bhosale, S. V. Chem. Rev. 2016, 116, 11685. (c) Das, A.; Ghosh, S. Chem. Commun. 2016, 52, 6860.

(3) Avlasevich, Y.; Li, C.; Müllen, K. J. Mater. Chem. 2010, 20, 3814.

(4) (a) Würthner, F.; Stolte, M. Chem. Commun. 2011, 47, 5109. (b) Zhan, X.; Facchetti, A.; Barlow, S.; Marks, T. J.; Ratner, M. A.; Wasielewski, M. R.; Marder, S. R. Adv. Mater. 2011, 23, 268. (c) Jones, B. A.; Facchetti, A.; Wasielewski, M. R.; Marks, T. J. J. Am. Chem. Soc. 2007, 129, 15259.

(5) Vollmann, H.; Becker, H.; Corell, M.; Streeck, H. Liebigs Ann. Chem. 1937, 531, 1.

(6) Thalacker, C.; Miura, A.; De Feyter, S.; De Schryver, F. C.; Würthner, F. Org. Biomol. Chem. 2005, 3, 414.

(7) (a) Thalacker, C.; Röger, C.; Würthner, F. J. Org. Chem. 2006, 71, 8098. (b) Shukla, J.; Mukhopadhyay, P. Eur. J. Org. Chem. 2019, 7770. (c) Gao, X.; Qiu, W.; Yang, X.; Liu, Y.; Wang, Y.; Zhang, H.; Qi, T.; Liu, Y.; Lu, K.; Du, C.; Shuai, Z.; Yu, G.; Zhu, D. Org. Lett. 2007, 9, 3917. (d) Kumar, Y.; Kumar, S.; Mandal, K.; Mukhopadhyay, P. Angew. Chem. Int. Ed. 2018, 57, 16318.

(8) Insuasty, A.; Maniam, S.; Langford, S. J. Chemistry 2019, 25, 7058.

(9) (a) Sakai, N.; Mareda, J.; Vauthey, E.; Matile, S. Chem. Commun. 2010, 46, 4225. (b) Kishore, R. S. K.; Kel, O.; Banerji, N.; Emery, D.; Bollot, G.; Mareda, J.; Gomez-Casado, A.; Jonkheijm, P.; Huskens, J.; Maroni, P.; Borkovec, M.; Vauthey, E.; Sakai, N.; Matile, S.J. Am. Chem. Soc. 2009, 131, 11106.

(10) Mas-Torrent, M.; Rovira, C. Chem. Rev. 2011, 111, 4833.

(11) (a) Andric, G.; Boas, J. F.; Bond, A. M.; Fallon, G. D.; Ghiggino, K. P.; Hogan, C. F.; Hutchison, J. A.; Lee, M. A.-P.; Langford, S. J.; Pilbrow, J. R.; Troup, G. J.; Woodward, C. P. Aust. J. Chem. 2004, 57, 1011. (b) Röger, C.; Würthner, F. J. Org. Chem. 2007, 72, 8070. (c) Röger, C.; Ahmed, S.; Würthner, F. Synthesis 2007, 12, 1872. (d) Bhosale, S. V.; Bhosale, S. V.; Bhargava, S. K. Org. Biomol. Chem. 2012, 10, 6455.

(12) (a) Facchetti, A. Chem. Mater. 2011, 23, 733. (b) Guo, X.; Kim, F. S.; Seger, M. J.; Jenekhe, S. A.; Watson, M. D. Chem. Mater. 2012, 24, 1434. 
(13) Shokouhi Mehr, H.; Romano, N. C.; Altamimi, R.; Modarelli, J. M.; Modarelli, D. A. Dalton Trans. 2015, 44, 3176.

(14) Sarkar, A.; Kölsch, J. C.; Berač, C. M.; Venugopal, A.; Sasmal, R.; Otter, R.; Besenius, P.; George, S. J. ChemistryOpen 2020, 9, 346.

(15) Kar, H.; Gehrig, D. W.; Allampally, N. K.; Fernández, G.; Laquai, F.; Ghosh, S. Chem. Sci. 2016, 7, 1115.

(16) Kar, H.; Ghosh, S. Chem. Commun. 2016, 52, 8818.

(17) Mukherjee, A.; Sakurai, T.; Seki, S.; Ghosh, S. Langmuir 2020, 36, 13096.

(18) Dhiman, S.; Ghosh, R.; George, S. J. ChemSystemsChem 2020, 2 , e1900042.

(19) Keene, F. Chirality in Supramolecular Assemblies: Causes and Consequences. Wiley: Chichester, 2016.

(20) Sarkar, A.; Dhiman, S.; Chalishazar, A.; George, S. J. Angew. Chem. Int. Ed. 2017, 56, 13767.

(21) Sethy, R.; Kumar, J.; Métivier, R.; Louis, M.; Nakatani, K.; Mecheri, N. M. T.; Subhakumari, A.; Thomas, K. G.; Kawai, T.; Nakashima, T. Angew. Chem. Int. Ed. 2017, 56, 15053.

(22) Yonezawa, S.; Sethy, R.; Fukuhara, G.; Kawai, T.; Nakashima, T. Chem. Commun. 2019, 55, 5793.

(23) Sethy, R.; Métivier, R.; Brosseau, A.; Kawai, T.; Nakashima, T. J. Phys. Chem. Lett. 2018, 9, 4516.

(24) Mukherjee, A.; Ghosh, S. Chemistry 2020, 26, 12874.

(25) Mukherjee, A.; Pal, D. S.; Kar, H.; Ghosh, S. Polym. Chem. 2020, 11, 7481.

(26) (a) Mukhopadhyay, R. D.; Ajayaghosh, A. Science 2015, 349, 241. (b) Sorrenti, A.; Leira-Iglesias, J.; Markvoort, A. J.; de Greef, T. F. A.; Hermans, T. M. Chem. Soc. Rev. 2017, 46, 5476. (c) Hartlieb, M.; Mansfield, E. D. H.; Perrier, S. Polym. Chem. 2020, 11, 1083. (d) Matern, J.; Dorca, Y.; Sánchez, L.; Fernández, G. Angew. Chem. Int. Ed. 2019, 58, 16730. (e) Wehner, M.; Würthner, F. Nat. Rev. Chem. 2020, 4, 38. (f) Ghosh, G.; Dey, P.; Ghosh, S. Chem. Commun. 2020, 56, 6757. (g) Weyandt, E.; Mabesoone, M. F. J.; de Windt, L. N. J.; Meijer, E. W.; Palmans, A. R. A.; Vantomme, G. Org. Mater. 2020, 2, 129.

(27) (a) Ogi, S.; Sugiyasu, K.; Manna, S.; Samitsu, S.; Takeuchi, M. Nat. Chem. 2014, 6, 188. (b) Ogi, S.; Stepanenko, V.; Thein, J.; Würthner, F. J. Am. Chem. Soc. 2016, 138, 670. (c) Greciano, E. E.; Matarranz, B.; Sánchez, L. Angew. Chem. Int. Ed. 2018, 57, 4697. (d) Jarrett-Wilkins, C.; He, X.; Symons, H. E.; Harniman, R. L.; Faul, C. F. J.; Manners, I. Chemistry 2018, 24, 15556. (e) Jung, S. H.; Bochicchio, D.; Pavan, G. M.; Takeuchi, M.; Sugiyasu, K. J. Am. Chem. Soc. 2018, 140, 10570. (f) Ogi, S.; Matsumoto, K.; Yamaguchi, S. Angew. Chem. Int. Ed. 2018, 57, 2339. (g) Chakraborty, A.; Ghosh, G.; Pal, D. S.; Varghese, S.; Ghosh, S. Chem. Sci. 2019, 10, 7345. (h) Wehner, M.; Röhr, M. I. S.; Stepanenko, V.; Würthner, F. Nat. Commun. 2020, 11, 5460. (i) Greciano, E. E.; Calbo, J.; Ortí, E.; Sánchez, L. Angew. Chem. Int. Ed. 2020, 59, 17517. (j) Matern, J.; Bäumer, N.; Fernández, G. J. Am. Chem. Soc. 2021, 143, 7164.

(28) Kar, H.; Gehrig, D. W.; Laquai, F.; Ghosh, S. Nanoscale 2015, 7, 6729.

(29) Kar, H.; Ghosh, G.; Ghosh, S. Chemistry 2017, 23, 10536.

(30) Ghosh, G.; Ghosh, S. Chem. Commun. 2018, 54, 5720.

(31) Sarkar, A.; Sasmal, R.; Empereur-Mot, C.; Bochicchio, D.; Kompella, S. V. K.; Sharma, K.; Dhiman, S.; Sundaram, B.; Agasti, S. S.; Pavan, G. M.; George, S. J. J. Am. Chem. Soc. 2020, 142, 7606.
(32) Sarkar, A.; Behera, T.; Sasmal, R.; Capelli, R.; Empereur-Mot, C.; Mahato, J.; Agasti, S. S.; Pavan, G. M.; Chowdhury, A.; George, S. J. J. Am. Chem. Soc. 2020, 142, 11528.

(33) (a) Poddar, M.; Sivakumar, G.; Misra, R. J. Mater. Chem. C 2019, 7, 14798. (b) Meher, N.; Iyer, P. K. Nanoscale 2019, 11, 13233. (c) Langdon-Jones, E. E.; Lloyd, D.; Hayes, A. J.; Wainwright, S. D.; Mottram, H. J.; Coles, S. J.; Horton, P. N.; Pope, S. J. A. Inorg. Chem. 2015, 54, 6606. (d) Greiner, R.; Schlücker, T.; Zgela, D.; Langhals, H. J. Mater. Chem. C 2016, 4, 11244. (e) Das, A.; Maity, B.; Koley, D.; Ghosh, S. Chem. Commun. 2013, 49, 5757.

(34) (a) Ghosh, S.; Pramanik, B.; Das, D. ChemNanoMat 2018, 4, 867. (b) Balachandra, C.; Govindaraju, T. J. Org. Chem. 2020, 85, 1525. (c) Jamadar, A.; Karan, C. K.; Roy, L.; Das, A. Langmuir 2020, 36, 3089.

(35) Chakraborty, S.; Varghese, S.; Ghosh, S. Chemistry 2019, 25, 16725.

(36) Dhbaibi, K.; Favereau, L.; Srebro-Hooper, M.; Quinton, C.; Vanthuyne, N.; Arrico, L.; Roisnel, T.; Jamoussi, B.; Poriel, C.; Cabanetos, C.; Autschbach, J.; Crassous, J. Chem. Sci. 2020, 11, 567.

(37) Tajima, K.; Fukui, N.; Shinokubo, H. Org. Lett. 2019, 21, 9516.

(38) (a) Jones, A. L.; Gish, M. K.; Zeman, C. J. IV; Papanikolas, J. M.; Schanze, K. S. J. Phys. Chem. A 2017, 121, 9579. (b) El-Khouly, M. E.; Moiseev, A. G.; van der Est, A.; Fukuzumi, S. ChemPhysChem 2012, 13, 1191. (c) Mishra, R.; Sujesh, S.; Archana, V. S.; Kaushik, A.; Gupta, G.; Singhal, R.; Sharma, G. D.; Sankar, J. Eur. J. Org. Chem. 2020, 1603. (d) Rundel, K.; Maniam, S.; Deshmukh, K.; Gann, E.; Prasad, S. K. K.; Hodgkiss, J. M.; Langford, S. J.; McNeill, C. R. J. Mater. Chem. A 2017, 5, 12266. (e) Kim, R.; Amegadze, P. S. K.; Kang, I.; Yun, H.-J.; Noh, Y.-Y.; Kwon, S.-K.; Kim, Y.-H. Adv. Funct. Mater. 2013, 23, 5719. (f) Guo, X.; Kim, F. S.; Seger, M. J.; Jenekhe, S. A.; Watson, M. D. Chem. Mater. 2012, 24, 1434. (g) Frischmann, P. D.; Mahata, K.; Würthner, F. Chem. Soc. Rev. 2013, 42, 1847.

(39) (a) Doria, F.; Folini, M.; Grande, V.; Cimino-Reale, G.; Zaffaroni, N.; Freccero, M. Org. Biomol. Chem. 2015, 13, 570. (b) Yildiz, U.; Coban, B. Appl. Biochem. Biotechnol. 2018, 186, 547. (c) Xu, J.-H.; Hou, Y.-M.; Ma, Q.-J.; Wu, X.-F.; Wei, X.-J. Spectrochim. Acta, Part A 2013, 112, 116. (d) Yuan, Y.; Xiao, Z.; Yang, B.; Huang, J.J. Mater. Chem. A 2014, 2, 6027. (e) Shi, K.; Zhang, F.; Di, C.-A.; Yan, T.-W.; Zou, Y.; Zhou, X.; Zhu, D.; Wang, J.-Y.; Pei, J. J. Am. Chem. Soc. 2015, 137, 6979. (f) Mann, J. L.; Yu, A. C.; Agmon, G.; Appel, E. A. Biomater. Sci. 2017, 6, 10. (g) Webber, M. J.; Appel, E. A.; Meijer, E. W.; Langer, R. Nat. Mater. 2016, 15, 13. (h) Zhao, X.; Liu, J.; Fan, J.; Chao, H.; Peng, X. Chem. Soc. Rev. 2021, 50, 4185.

(40) (a) Tan, L.; Guo, Y.; Yang, Y.; Zhang, G.; Zhang, D.; Yu, G.; Xu, W.; Liu, Y. Chem. Sci. 2012, 3, 2530. (b) Luo, H.; Cai, Z.; Tan, L.; Guo, Y.; Yang, G.; Liu, Z.; Zhang, G.; Zhang, D.; Xu, W.; Liu, Y. J. Mater. Chem. C 2013, 1, 2688. (c) Zhao, Y.; Guo, Y.; Liu, Y. Adv. Mater. 2013, 25, 5372. (d) Stalder, R.; Puniredd, S. R.; Hansen, M. R.; Koldemir, U.; Grand, C.; Zajaczkowski, W.; Müllen, K.; Pisula, W.; Reynolds, J. R. Chem. Mater. 2016, 28, 1286. (e) Riaño Carnerero, A.; López Espejo, G.; Mancheño Real, M. J.; Eckstein, B.; González-Cano, R. C.; Melkonyan, F. S.; Facchetti, A.; Marks, T. J.; Casado, J.; López Navarrete, J. T.; Segura, J. L.; Ponce Ortiz, R. J. Mater. Chem. C 2017, 5, 9439. (f) Lin, Z.; Chen, L.; Xu, Q.; Shao, G.; Zeng, Z.; Wu, D.; Xia, J. Org. Lett. 2020, 22, 2553. 